\title{
The cuticular wax inhibitor locus Iw2 in wild diploid wheat Aegilops tauschii: phenotypic survey, genetic analysis, and implications for the evolution of common wheat
}

\author{
Ryo Nishijima', Julio C M lehisa', Yoshihiro Matsuoka² and Shigeo Takumi*
}

\begin{abstract}
Background: Cuticular wax production on plant surfaces confers a glaucous appearance and plays important roles in plant stress tolerance. Most common wheat cultivars, which are hexaploid, and most tetraploid wheat cultivars are glaucous; in contrast, a wild wheat progenitor, Aegilops tauschii, can be glaucous or non-glaucous. A dominant non-glaucous allele, Iw2, resides on the short arm of chromosome 2D, which was inherited from Ae. tauschii through polyploidization. IW2 is one of the major causal genes related to variation in glaucousness among hexaploid wheat. Detailed genetic and phylogeographic knowledge of the Iw2 locus in Ae. tauschii may provide important information and lead to a better understanding of the evolution of common wheat.
\end{abstract}

Results: Glaucous Ae. tauschii accessions were collected from a broad area ranging from Armenia to the southwestern coastal part of the Caspian Sea. Linkage analyses with five mapping populations showed that the glaucous versus non-glaucous difference was mainly controlled by the Iw2 locus in Ae. tauschii. Comparative genomic analysis of barley and Ae. tauschii was then used to develop molecular markers tightly linked with Ae. tauschii Iw2. Chromosomal synteny around the orthologous IW2 regions indicated that some chromosomal rearrangement had occurred during the genetic divergence leading to Ae. tauschii, barley, and Brachypodium. Genetic associations between specific Iw2-linked markers and respective glaucous phenotypes in Ae. tauschii indicated that at least two non-glaucous accessions might carry other glaucousness-determining loci outside of the IW2 locus.

Conclusion: Allelic differences at the Iw2 locus were the main contributors to the phenotypic difference between the glaucous and non-glaucous accessions of Ae. tauschii. Our results supported the previous assumption that the D-genome donor of common wheat could have been any Ae. tauschii variant that carried the recessive iw2 allele.

Keywords: Allopolyploid speciation, Cuticluar wax inhibitor, Synthetic wheat, Wheat evolution

\section{Background}

Cuticular wax production on aerial surfaces of plants has important roles in various physiological functions and developmental events; the wax prevents non-stomatal water loss, inhibits organ fusion during development, protects from UV radiation damage, and imposes a physical barrier against pathogenic infection [1-4]. The trait, the coating of leaf and stem surfaces with a waxy

\footnotetext{
*Correspondence: takumi@kobe-u.ac.jp

${ }^{1}$ Graduate School of Agricultural Science, Kobe University, Rokkodai 1-1,

Nada, Kobe 657-8501, Japan

Full list of author information is available at the end of the article
}

whitish substance, is called glaucousness. In common wheat (Triticum aestivum L., $2 \mathrm{n}=6 \mathrm{x}=42$, genome constitution BBAADD), dominant alleles $W 1$ and $W 2$, control the wax production and have been assigned to chromosomes $2 \mathrm{~B}$ and $2 \mathrm{D}$, respectively [5,6]. Additionally, dominant homoeoalleles for non-glaucousness, $I w 1$ and $I w 2$, have also been mapped to the short arms of chromosomes $2 \mathrm{~B}$ and 2D, respectively [6-9]. Wheat plants with either the $w 1, w 2, I w 1$ or $I w 2$ allele show the non-glaucous phenotype, indicating that $W 1$ and $W 2$ are functionally redundant for the glaucous phenotype and that a single Iw dominant allele is sufficient to inhibit the glaucous 
phenotype even in the presence of a $W 1$ or $W 2$ allele $[3,6]$. Wax composition in wheat plants with one $I w$ dominant allele is biochemically different from that in glaucous plants of any genotype; $ß$-diketones are completely absent from extracts of cuticular wax from $I w$ plants, while aldehydes and primary alcohols are very abundant in these extracts $[3,10]$. A fine map around the Iw 1 region on $2 \mathrm{BS}$ was constructed using an $\mathrm{F}_{2}$ population of tetraploid wheat (Triticum turgidum L., $2 \mathrm{n}=4 \mathrm{x}=28, \mathrm{BBAA}$ ), and three markers tightly linked to $I w 1$ were developed [10,11]. A high-resolution map of Iw 2 on 2DS has been developed in hexaploid wheat, and two markers tightly linked to $I w 2$ were also developed [11]. Comparative mapping of $I w 1$ and $I w 2$ shows that the two loci are homoeologous to each other and orthologous to the same chromosomal region of Brachypodium distachyon (L.) P. Beauv. [11]. Recently, a third wax-inhibitor locus Iw3 was identified on chromosome 1BS from wild emmer wheat [12], and a fine map of the Iw3 locus is available [13]. Iw2 is located on 2DS in Aegilops tauschii Coss. ( $2 \mathrm{n}=2 \mathrm{x}=14$, DD), which is diploid and the progenitor of the D-genome of common wheat [14], but to our knowledge, a high-resolution genetic map of the Iw2 region in Ae. tauschii has not been constructed.

Common wheat is an allohexaploid species derived from interspecific hybridization between tetraploid wheat with a BBAA genome and Ae. tauschii. Most cultivated varieties of tetraploid wheat are glaucous, even though non-glaucous types are frequently found among wild tetraploid accessions $[6,15]$; this variation indicates that the glaucous phenotype might have been a target of artificial selection during the domestication of tetraploid wheat. Glaucous accessions of Ae. tauschii are found in the area ranging from Transcaucasia to the southern coastal region of the Caspian Sea $[5,16]$. Almost all varieties of common wheat carry $W 1$ and $W 2$ and lack $I w 1$ and $I w 2$; therefore, the D-genome donor of common wheat is assumed to have had the recessive $i w 2$ allele [5]. Glaucous Ae. tauschii accessions have the $W 2$ and $i w 2$ alleles. Non-glaucous accessions of Ae. tauschii that have the W2 and Iw2 alleles have been recovered from a wide distribution range in central Eurasia [5]. Moreover, discovery of a non-glaucous Ae. tauschii accession with the $w 2$ recessive allele has not yet been reported.

Therefore, analysis of the Iw2 locus may provide important information that improves our understanding of the evolution of common wheat. Population structure analyses of Ae. tauschii indicate that the whole species Ae. tauschii can be divided into three major genealogical lineages, tauschii lineage 1 (TauL1), TauL2, and TauL3, and that genetically genomes of TauL2 accessions are most closely related to the D genome of common wheat [17-19]. Recently, a whole-genome shotgun strategy was used to generate a draft genome sequence of Ae. tauschii that has been published; this draft anchors $1.72 \mathrm{~Gb}$ of the $4.36 \mathrm{~Gb}$ genome to chromosomes [20]. A physical map of the Ae. tauschii genome that covers $4 \mathrm{~Gb}$ is also available [21]. The objectives of this study were (1) to examine the natural variation in glaucousness among a species-wide set of Ae. tauschii accessions, (2) to use $\mathrm{F}_{2}$ populations of Ae. tauschii accessions and synthetic hexaploid wheat lines to fine-map Iw2 locus on 2DS, (3) to develop molecular markers that are closely linked to Iw2 based on chromosomal synteny between barley and wheat chromosomes, and (4) to provide novel insights into the evolutionary relationship between the Ae. tauschii genome and the $\mathrm{D}$ genome of common wheat on the basis of the detailed genetic and phylogeographic knowledge of the Iw 2 chromosomal region.

\section{Methods}

\section{Plant materials and phenotype evaluation}

In all, 210 Ae. tauschii accessions were used in this study [22]. Their passport data, including geographical coordinates, have been provided in previous reports [23,24]. Previously, 206 of the Ae. tasuchii accessions were grouped into the three lineages, TauL1, TauL2, and TauL3, based on DArT marker genotyping analysis [19]. Of the 210 accessions, 12 were previously identified as subspecies strangulata based on the sensu-strico criteria $[25,26]$. Seeds from two Ae. tauschii hybrid $\mathrm{F}_{2}$ populations $(\mathrm{n}=116$ from each population) were sown in November 2011; one $\mathrm{F}_{2}$ population resulted from a cross between KU-2154 (non-glaucous) and KU-2126 (glaucous), the other from a KU-2003 (non-glaucous) by KU-2124 (glaucous) cross. In the 2012-2013 season, 169 additional $F_{2}$ individuals of the KU-2154/KU-2126 population were grown to increase the size of the mapping population.

Previously, 82 synthetic hexaploid wheat lines were produced from crosses between a tetraploid wheat ( $T$. turgidum subspecies durum (Desf.) Husn.) cultivar Langdon (Ldn) and 69 Ae. tauschii accessions [26,27]. These synthetic hexaploid wheat lines were used for crossing and phenotypic studies conducted in a glasshouse at Kobe University. Ldn shows the glaucous phenotype and is homozygous for the $i w 1$ allele [10]. Each synthetic hexaploid thus contained the A and B genomes from Ldn and one of many diverse $\mathrm{D}$ genomes originating from the Ae. tauschii pollen parents. In the present study, four $F_{3}$ plants derived from one $F_{2}$ plant of each synthetic hexaploid were grown individually during the 2007-2008 season in pots that were arranged randomly in the glasshouse; these $276 \mathrm{~F}_{3}$ plants were used for crossing and phenotypic observation. The following three pairs of synthetic hexaploids were used to generate three $\mathrm{F}_{2}$ mapping populations: Ldn/PI476874 (non-glaucous) and Ldn/ KU-2069 (glaucous), Ldn/IG126387 (non-glaucous) and Ldn/KU-2159 (glaucous), and Ldn/KU-2124 (glaucous) 
and Ldn/IG47259 (non-glaucous). The first population (Ldn/PI476874//Ldn/KU-2069) comprised $106 \mathrm{~F}_{2}$ individuals grown in the glasshouse during the 2009-2010 season. Seeds from the other two populations were sown in November 2011, with the numbers of individuals in each being 100 (Ldn/KU-2159//Ldn/IG126387) and 82 (Ldn/KU-2124//Ldn/IG47259).

For analysis of the $\mathrm{D}$ genome of common wheat, 17 landraces collected in Iran were supplied from the National BioResource Project (NBRP) KOMUGI (http://www.shigen. nig.ac.jp/wheat/komugi). These Iranian landraces-KU3097, KU-3098, KU-3121, KU-3126, KU-3136, KU-3162, KU-3184, KU-3189, KU-3202, KU-3232, KU-3236, KU-3274, KU-3289, KU-10393, KU-10439, KU-10480, and KU-10510-each showed the glaucous phenotype.

Glaucousness was evaluated based on the presence or absence of wax production on the surface of peduncles and spikes in both Ae. tauschii and synthetics. Wax production was clearly visible and whitish.

\section{Genotyping and construction of linkage maps}

To amplify PCR fragments containing molecular markers, some of which were simple sequence repeats (SSRs), total DNA was extracted from leaves of the parental strains and $\mathrm{F}_{2}$ individuals. For SSR genotyping, 40 cycles of PCR were performed using 2x Quick Taq HS DyeMix (TOYOBO, Osaka, Japan) and the following conditions: $10 \mathrm{~s}$ at $94^{\circ} \mathrm{C}$, $30 \mathrm{~s}$ at the appropriate annealing temperature $(72,73$, or $75^{\circ} \mathrm{C}$ ), and $30 \mathrm{~s}$ at $68^{\circ} \mathrm{C}$. The last step was a $1-\mathrm{min}$ incubation at $68^{\circ} \mathrm{C}$. Information on SSR markers and the respective annealing temperatures was obtained from the NBRP KOMUGI web site (http://www.shigen.nig.ac. jp/wheat/komugi/strains/aboutNbrpMarker.jsp) and the GrainGenes web site (http://wheat.pw.usda.gov/GG2/maps. shtml). PCR products were resolved in $2 \%$ agarose or $13 \%$ nondenaturing polyacrylamide gels and visualized under UV light after staining with ethidium bromide. The MAPMAKER/EXP version 3.0b package was used for genetic mapping [28]. The threshold for log-likelihood scores was set at 3.0, and genetic distances were calculated with the Kosambi function [29].

Each polymorphism at the Ppd-D1 locus on 2DS was detected with allele-specific primers and methodology described by Beales et al. [30]. A common forward primer, Ppd-D1_F (5'-ACGCCTCCCACTACACTG-3'), and two reverse primers, Ppd-D1_R1 (5'-GTTGGTTCAAACAG AGAGC-3') and Ppd-D1_R2 (5'-CACTGGTGGTAGCT GAGATT-3'), were used for this PCR analysis. PCR products amplified with Ppd-D1_F and Ppd-D1_R2 detected a 2,089-bp deletion in the $5^{\prime}$ upstream region of $P p d-D 1$ that is indicative of the photoperiod-insensitive Ppd-D1a allele [30]. EST-derived sequence-tagged site (STS) markers on 2DS, TE6, and WE6 were also used for genotyping; two STS markers for each locus, and these markers were previously developed along with the Iw2-linked markers [7]. The amplified PCR products were separated via electrophoresis through a $2 \%$ agarose or $13 \%$ nondenaturing polyacrylamide gel and then stained with ethidium bromide.

\section{Development of additional markers linked to Iw2}

In our previous studies, we conducted deep-sequencing analyses of the leaf and spike transcriptomes of two $A e$. tauschii accessions that represented two major lineages, and discovered more than 16,000 high-confidence single nucleotide polymorphisms (SNPs) in 5,808 contigs [31,32]. Contigs with the SNPs were searched with blastn against Ae. tauschii genome sequences [20] and barley genome sequences [33]; these genome sequences included highconfidence genes with an $E$-value threshold of $10^{-5}$ and hit length $\geq 50 \mathrm{bp}$, fingerprinted contigs, and whole genome shotgun assemblies.

To choose scaffolds for Ae. tauschii sequences throughout the Iw 2 chromosomal region, all the genes contained in each scaffold were searched with blastn against the barley genomic sequence using parameters described above. Scaffolds containing at least one gene aligned on the distal region of chromosome 2HS (between $3.66 \mathrm{Mb}$ and $5.51 \mathrm{Mb}$ ) were considered possible candidates for marker development. Scaffolds without genes were anchored based on respective results from the blastn searches against the barley genome. First, high-confidence SNPs [31,32] plotted in this 2HS chromosomal segment were used for marker development to refine the target region. Next, SciRoKo version 3.4 [34] was used with search mode setting "mismatched; fixed penalty" to identify additional SSR markers in sequence data of candidate scaffolds. Additional SNPs were also identified on candidate scaffolds by sequencing approximately 700 bp of amplified DNA of two Ae. tauschii accessions, KU-2154 and KU-2126. The nucleotide sequences were determined using an Applied Biosystems 3730xl DNA Analyzer (Applied Biosystems, Foster City, CA, USA), and SNPs were found via sequence alignments constructed and searched with GENETYX-MAC version 12.00 software (Whitehead Institute for Biomedical Research, Cambridge, MA, USA).

For genotyping, total DNA was extracted from leaves taken from each of the 210 Ae. tauschii accessions and the 17 Iranian wheat landraces. SSR amplification and detection of polymorphisms at these loci were conducted as described above. The identified SNPs were then further developed into cleaved amplified polymorphic sequence (CAPS) or high resolution melting (HRM) markers. The primer sequences for each SNP marker and any relevant restriction enzymes are summarized in Additional file 1. PCR and subsequent analyses were performed as described previously [31,32,35]. 


\section{Blast analysis of the Ae. tauschii genes relative to the Brachypodium genome}

Nucleotide sequences and annotation information of the selected Ae. tauschii scaffolds were analyzed with reference to the Ae. tauschii draft genome data, which was published by Jia et al. [20]. Reference sequences from Brachypodium [36] were searched against the National Center for Biotechnology Information (NCBI) NR protein database using the blastx algorithm with an $E$-value cut-off of $10^{-3}$.

\section{Association analysis of the linked markers with glaucousness}

The $\mathrm{Q}+\mathrm{K}$ method was conducted using a mixed linear model (MLM) function in TASSEL ver 4.0 software [37] for an association analysis by incorporating phenotypic and genotypic data and information on population structure. In a previous report, the Bayesian clustering approach implemented in the software program STRUCTURE 2.3 [38] was used with the setting $k=2$ to predict the population structure of the Ae. tauschii accessions [19]. The Q-matrix of population membership probabilities was served as covariates in MLM. Kinship (K) was calculated in TASSEL based on the genotyping information of the 169 DArT markers for the 206 Ae. tauschii accessions [19]. We performed the $F$-statistics and calculated the $P$-values for the $F$-test, and the threshold value was set as $1 \mathrm{E}-3$ for the significant association. We omitted the target markers from the association analysis when their minor allele frequencies were less than 0.05 .

\section{Results}

Wax production variation among Ae. tauschii accessions and among synthetic wheat lines

Of the 210 Ae. tauschii accessions examined, only 20 (9.5\%) exhibited the glaucous phenotype and produced whitish wax on the surfaces of peduncles and spikes (Figure 1A-D, Additional file 2). Wax production for each accession was completely consistent between the Fukui and Kobe environments. Each glaucous accession belonged to Ae. tauschii subspecies tauschii; in other words, none belonged to Ae. tauschii subspecies strangulata; the geographic distribution of glaucous accessions was limited to the area that spans from Transcaucasia to the southern coastal region of the Caspian Sea (Figure $1 \mathrm{H}$ ). In the eastern habitats (central Asia, Afghanistan, Pakistan, India, and China) of the species range, no glaucous accession was found. Of the 20 glaucous accessions, 19 belonged to the TauL2 lineage, and only one (IG127015 collected in Armenia) belonged to the TauL1 lineage (Additional file 2).

Of the 82 synthetic wheat lines that we examined, 15 exhibited whitish wax production on the peduncle and spike surface (Figure 1E-G), whereas no wax production was evident in any of the 67 other lines (Additional file 2). Of the 15 lines that showed the glaucous phenotype, 13 were produced by crossing Ldn with glaucous Ae. tauschii accessions, and each of the 67 non-glaucous lines was produced by crossing Ldn with a non-glaucous Ae. tauschii accession. Notably, two synthetic lines, Ldn/KU-2104 and Ldn/KU-2105, exhibited the glaucous phenotype even though their parental Ae. tauschii accessions were non-glaucous.

Mapping of the Iw2 locus in Ae. tauschii and synthetic wheat Two $\mathrm{F}_{2}$ populations of Ae. tauschii and three $\mathrm{F}_{2}$ populations from the synthetic wheat lines were analyzed to map the loci that control inhibition of wax production. Each $F_{1}$ plant used for the five cross combinations exhibited the non-glaucous phenotype. In each $\mathrm{F}_{2}$ population, the ratio of non-glaucous to glaucous individuals was 3:1; these findings were statistically significant and consistent with Mendelian segregation of alleles of a single gene (Table 1). These results indicated that a single genetic locus was associated with the phenotypic difference between nonglaucous and glaucous surfaces on peduncles and spikes, and that allele conferring the non-glaucous phenotype was dominant and the allele conferring the glaucous phenotype was recessive.

A single locus that controlled inhibition of wax production in Ae. tauschii was mapped to the same region of the short arm of chromosome 2D in each $\mathrm{F}_{2}$ mapping population (Figure 2). In the KU-2003/KU-2124 population, the locus that controlled inhibition of wax production, together with the loci for 25 SSR markers and Ppd-D1, was assigned to chromosome $2 \mathrm{D}$, and the map length was $230.0 \mathrm{cM}$ with an average inter-loci interval of $8.85 \mathrm{cM}$. In the KU-2154/ KU-2126 population, the locus that controlled inhibition of wax production, together with 14 SSR and 2 STS markers and Ppd-D1, was assigned to chromosome 2D, and the map length was $175.4 \mathrm{cM}$ with average inter-loci spacing of $10.32 \mathrm{cM}$. In the three synthetic wheat populations, Ldn/ KU-2159//Ldn/IG126387, Ldn/KU-2124//Ldn/IG47259, and Ldn/PI476874//Ldn/KU-2069, the locus that controlled inhibition of wax production was mapped to a similar position on the short arm of chromosome 2D (Figure 2). In these three synthetic wheat populations, the locus that controlled inhibition of wax production was mapped together with 11 to 13 SSR markers, 0 to 2 STS markers, and Ppd-D1; additionally, the map lengths ranged from 79.4 to $93.8 \mathrm{cM}$ with an average inter-loci spacing of 4.96 to $8.53 \mathrm{cM}$.

WE6 and TE6 are EST-derived STS markers that are linked to $I w 2$ in two mapping populations $[7,9]$. In three of our mapping populations, linkage of the non-glaucousness loci to WE6 and TE6 were confirmed. Thus, the position of one locus that controlled inhibition of wax production in Ae. tauschii corresponded to the well-known wax inhibitor 


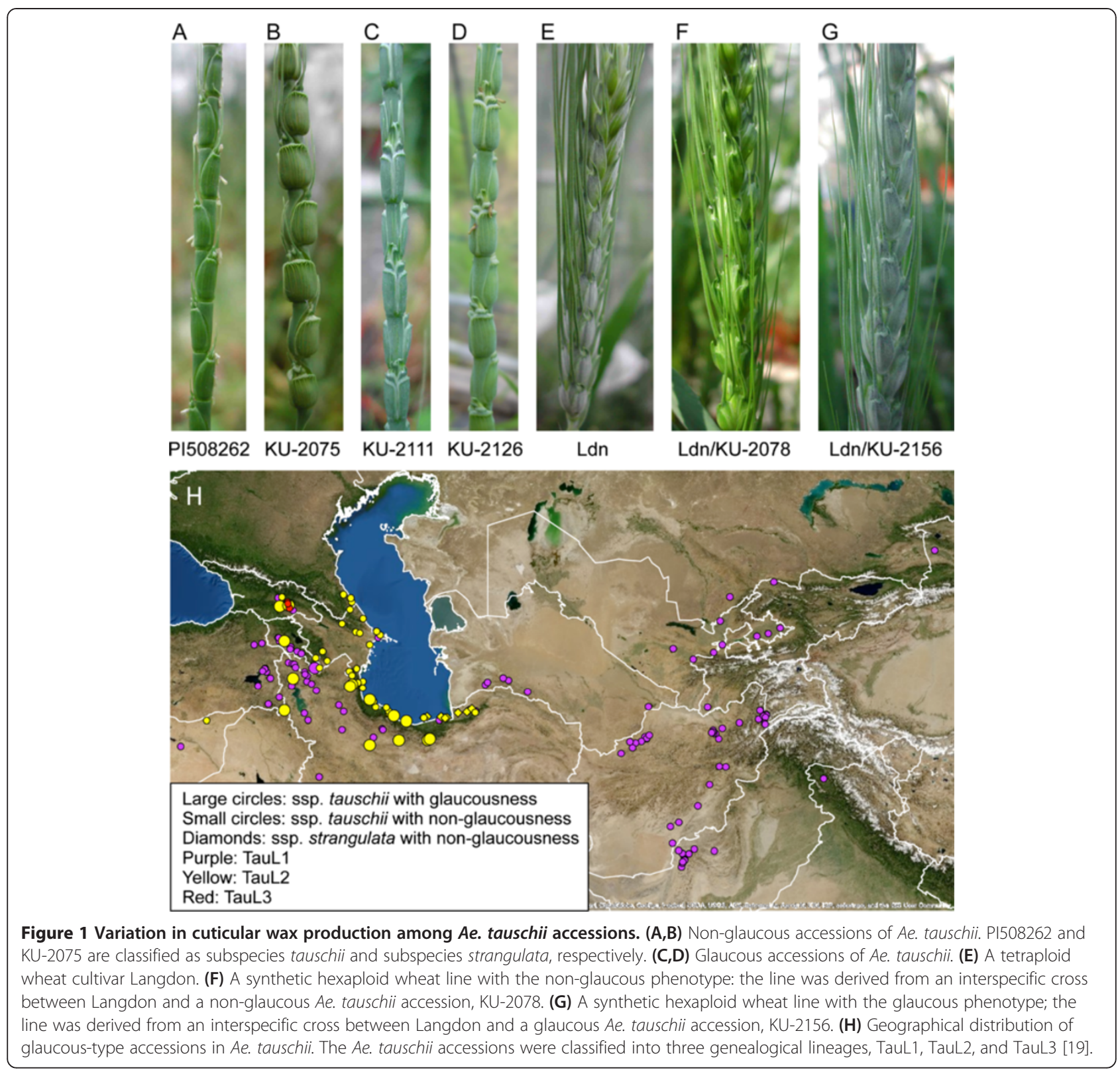

Table 1 Segregation analysis of the non-glaucous phenotype in the five $F_{2}$ mapping populations

\begin{tabular}{|c|c|c|c|c|c|}
\hline$F_{2}$ population & $N$ & Non-glaucous type & Glaucous type & $\mathrm{x}^{2}$ value* & $P$ value \\
\hline KU-2003/KU-2124 & 116 & 89 & 27 & 0.184 & 0.668 \\
\hline KU-2154/KU-2126 & 116 & 78 & 38 & 3.724 & 0.054 \\
\hline Ldn/KU-2159//Ldn/IG126387 & 100 & 71 & 29 & 0.853 & 0.356 \\
\hline Ldn/KU-2124//Ldn/IG47259 & 82 & 65 & 17 & 0.797 & 0.372 \\
\hline Ldn/PI476874//Ldn/KU-2069 & 106 & 77 & 29 & 0.314 & 0.575 \\
\hline
\end{tabular}

*Expected segregation ratio was 3:1. 


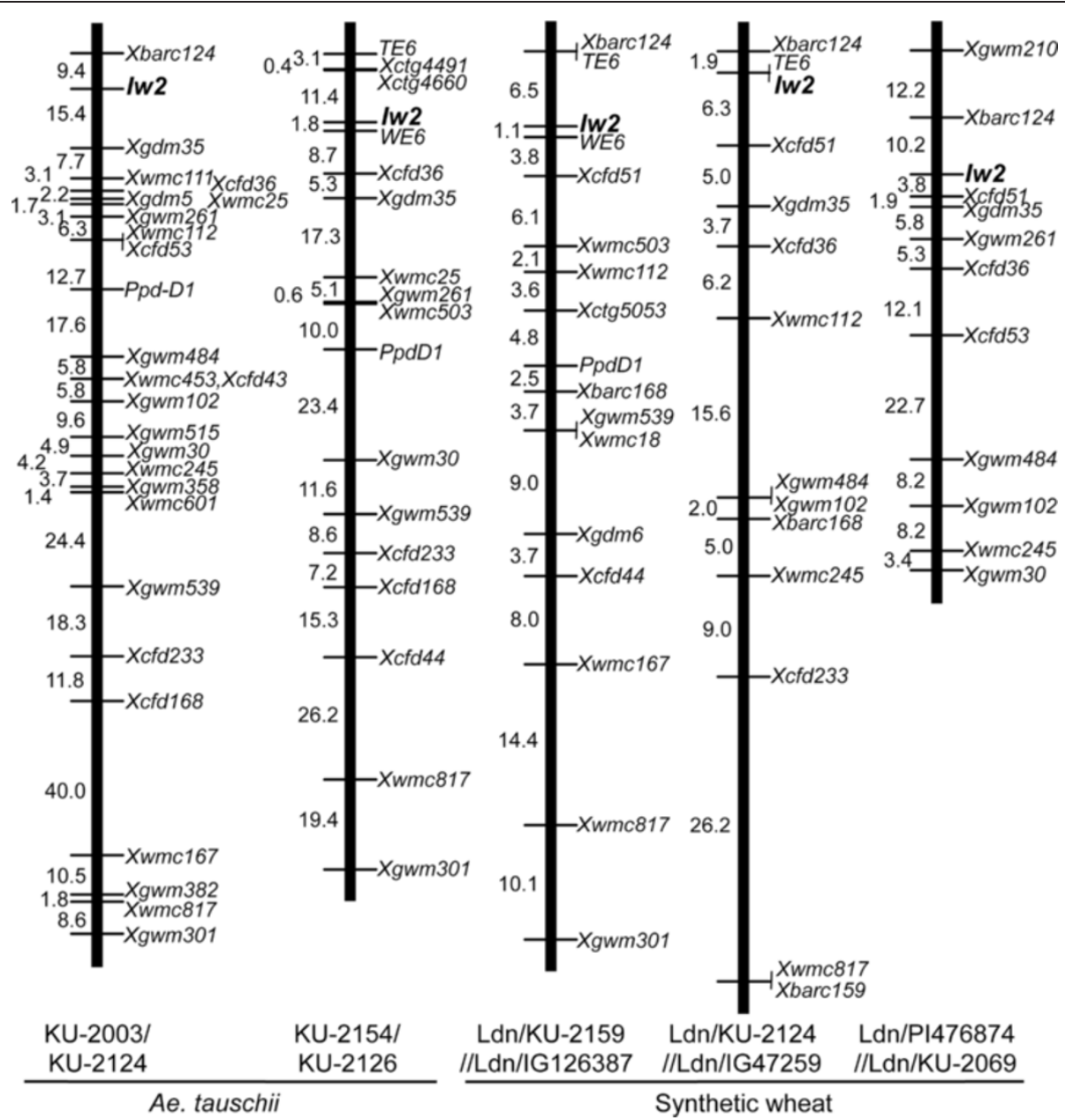

Figure $\mathbf{2}$ Linkage maps of Iw2 on chromosome 2D. Two and three mapping populations were generated for Ae. tauschii and synthetic hexaploid wheat, respectively. Genetic distances are represented in centimorgans to the left of each chromosome.

gene, $I w 2$, on chromosome 2D [6,7]. Therefore, hereafter, all glaucousness-related loci mapped in this study were considered to be identical to $I w 2$.

\section{Fine mapping of the Iw2 locus}

The high-confidence SNPs derived from Ae. tauschii RNAseq data have been plotted onto barley chromosomes [32], and physical map information for the barley genome is available [33]. Additionally, physical map information for Ae. tauschii and 16,876 scaffolds that constitute $1.49 \mathrm{~Gb}$ from the draft Ae. tauschii genome sequence are anchored to the Ae. tauschii linkage map [20,21]. The RNA-seqderived SNP information [31,32] was used to map seven high-confidence SNPs, represented as Xctg loci in Figure 3, throughout the Iw 2 chromosomal region in the KU-2154/ KU-2126 $F_{2}$ population. Of the seven Xctg loci, four were located within the $8.8 \mathrm{cM}$ chromosomal region immediately surrounding $I w 2$. Nucleotide sequences of the four cDNAs corresponding to these Xctg loci were used as queries to select the carrier scaffolds from Ae. tauschii sequences.
We selected the Ae. tauschii scaffolds that mapped near the Xctg-carrying Ae. tauschii scaffolds based on synteny between the wheat and barley genomes and the barley physical map [39]. In all, 18 Ae tauschii scaffolds were assigned in silico to an area of the Ae. tauschii genome that corresponded to the $I w 2$ region in the physical map of barley chromosome $2 \mathrm{H}$ (Figure 3 ). Using a previously developed physical map of the Ae. tauschii 2DS chromosome [21], we mapped six Ae. tauschii scaffolds in silico to the corresponding region in the 2DS physical map. Nucleotide sequences of the selected scaffolds were used to design CAPS or SSR markers for each scaffold, and the markers that were polymorphic between KU-2154 and KU2126 were then mapped in the $F_{2}$ population (Figure 3 ).

Of the selected scaffolds, 23 were mapped to the $I w 2$ chromosomal region on 2DS, and the remaining three scaffolds were assigned to other chromosomes. In the KU-2154/KU-2126 population with $115 \mathrm{~F}_{2}$ individuals, the Iw2 locus was mapped within the $1.1 \mathrm{cM}$ interval between the most closely linked markers (Figure 3). A dominant 


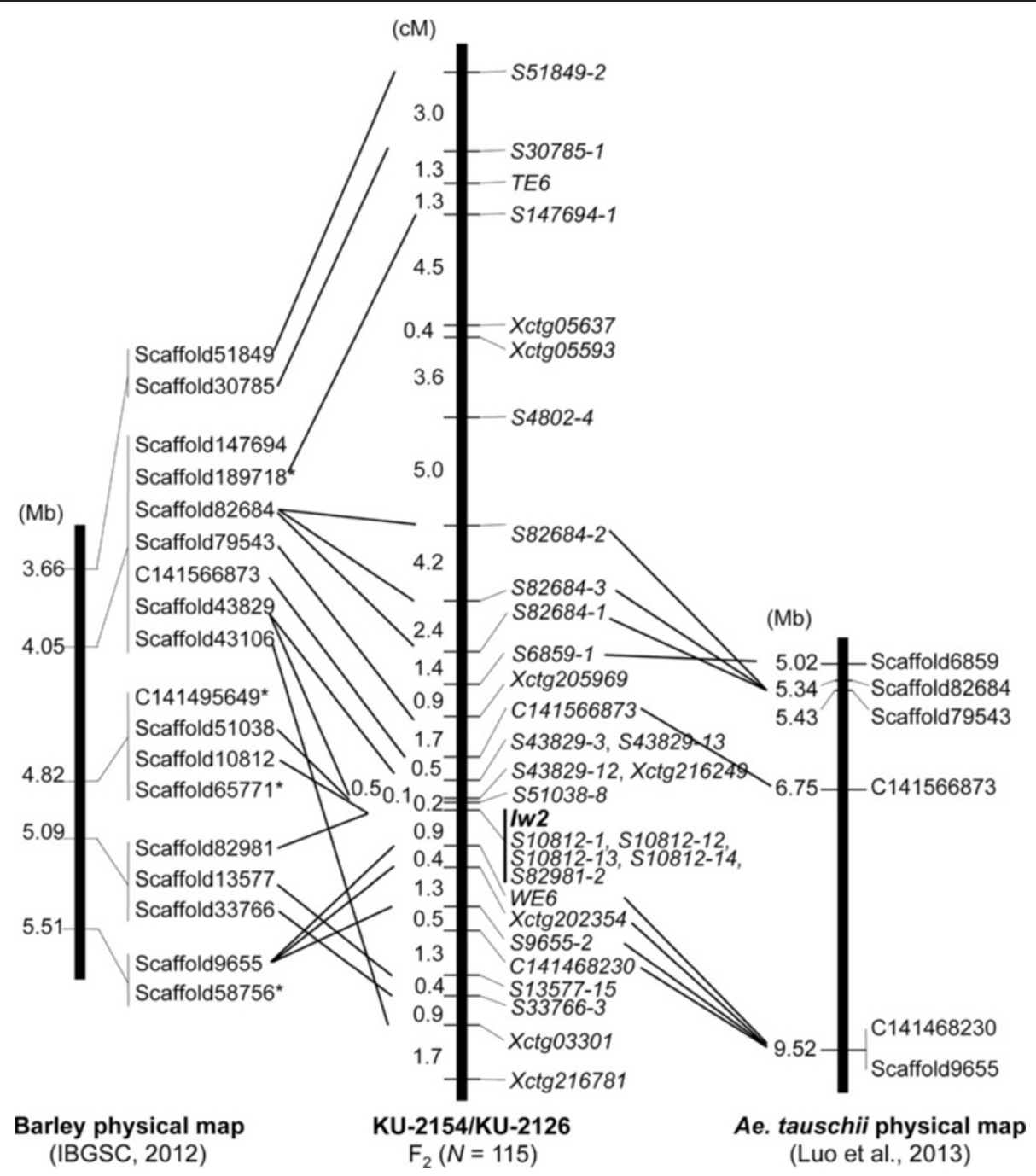

Figure 3 Comparison of the Iw2 linkage map, which contains the Ae.tauschii scaffolds, with the physical maps of barley and Ae. tauschii. The Ae. tauschii scaffolds were assigned to regions of the barley physical map of chromosome $2 \mathrm{H}$ [33]. An Ae. tauschii physical map with the mapped scaffolds [21] is represented. Scaffold positions (Mb) and numbers [20,21] are shown on the left and right of each chromosome, respectively.

marker (S51038-8), derived from the Ae. tauschii scaffold 51038 sequence, was located $0.2 \mathrm{cM}$ distal to $I w 2$, and the WE6 SSR marker was located $0.9 \mathrm{cM}$ proximal to Iw2. Five co-dominant markers, derived from two Ae. tauschii scaffolds 10812 and 82981, co-localized with Iw2. The marker order in the KU-2154/KU-2126 linkage map was generally conserved with that in the barley $2 \mathrm{H}$ physical map. However, barley scaffold 9655 was more closely linked to the barley $I w 2$ ortholog than were two corresponding Ae. tauschii scaffolds, 13577 and 33766, to the tauschii Iw2 ortholog; this positioning indicated that a local inversion had occurred in the region proximal to $I w 2$ during the divergence between barley and tauschii.

Next, $F_{2}$ individuals of the KU-2154/KU-2126 population and 12 markers from five Ae. tauschii scaffolds were used to construct a fine map of $I w 2$ (Figure 4A). Based on this linkage map, $I w 2$ was located within the $0.7 \mathrm{cM}$ between Xctg216249/S51038-8 and WE6 and co-localized with five markers derived from two scaffolds, 10812 and 82981. Each of the five scaffolds was 63 to $334 \mathrm{~kb}$ in length and included one to 16 putative protein-coding genes [20,21]; marker positions of each scaffold are indicated in Figure 4B. Of the 12 markers, eight were derived from intergenic regions, the other four from open reading frames.

In all, 36 genes were evident on the five scaffolds, and gene annotation could be confirmed for 27 of the 36 genes (Table 2). Of these 27 Ae. tauschii genes, 10 putatively encoded cytochrome P450 monooxygenase proteins, and eight encoded disease-related proteins. Additionally, genes encoding laccase, agmatine coumaroyltransferase, receptor 


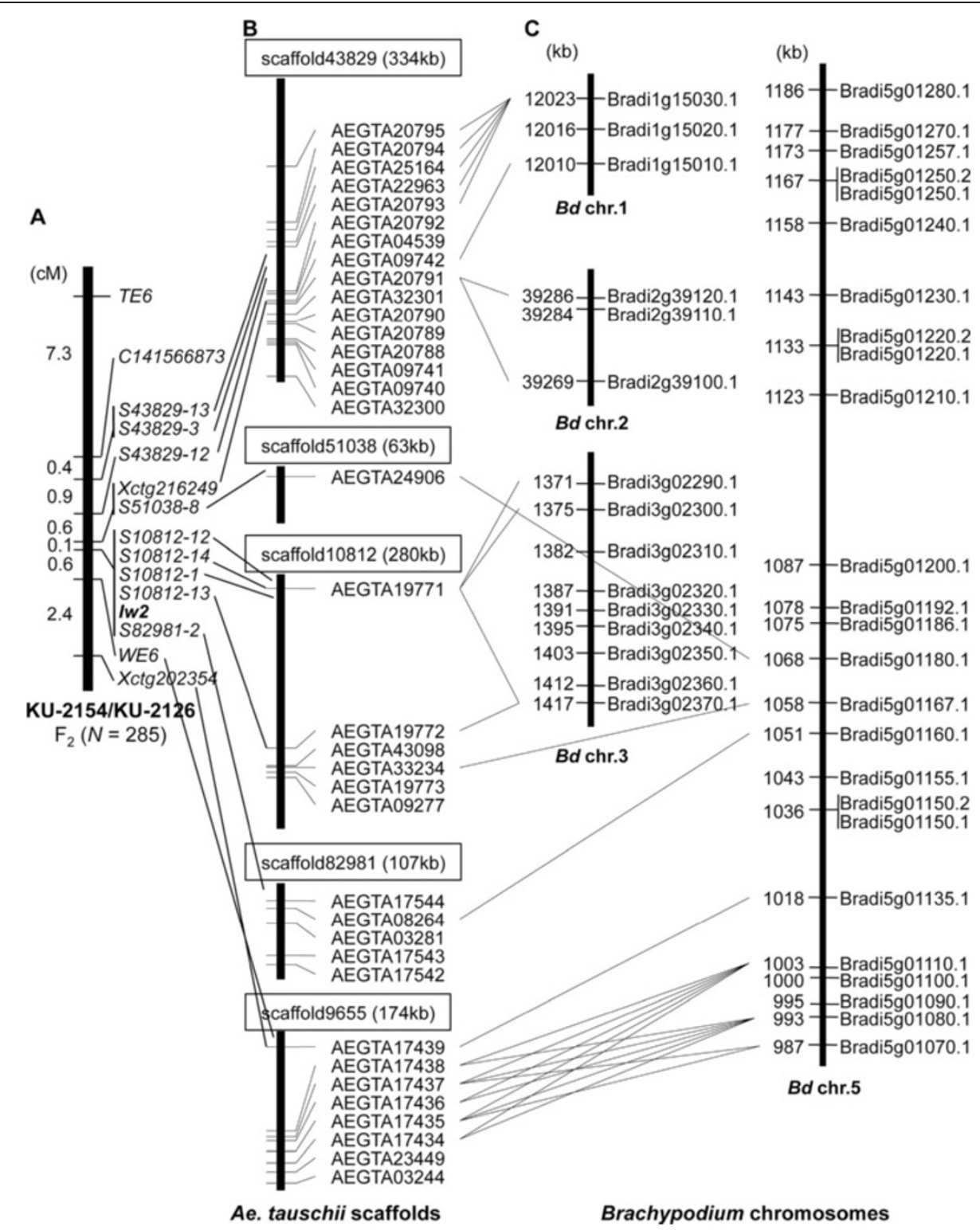

Figure 4 Assignment of protein-encoding genes found on the scaffolds around Iw2 to orthologs on Brachypodium chromosomes. (A) Linkage map of the region around IW2 generated with $285 \mathrm{~F}_{2}$ individuals. Genetic distances (CM) are shown on the left, and markers on the right. (B) The figure shows the positions of putative genes and mapped markers in the Ae. tauschii scaffolds anchored to the IW2 region. (C) The IW2-orthologous regions on Brachypodium chromosomes based on the blastx search of anchored Ae. tauschii genes. Brachypodium genes are shown on the right, and their position (kb) on the left.

kinase, and cell number regulator 2-like were found on the two scaffolds that co-localized with $I w 2$.

The Ae. tauschii scaffolds that included protein coding genes were used as queries to search the Brachypodium genomic information via a blastn search. Of the Ae. tauschii genes on the five scaffolds, 18 had obvious orthologs in the Brachypodium genome (Figure 4C). Putative orthologs of the Ae. tauschii genes from the four scaffolds were assigned to the 987 to $1068 \mathrm{~kb}$ region of Brachypodium chromosome 5. In addition, three Brachypodium paralogs (Bradi5g01220.1, Bradi5g01220.2, and Bradi5g01230.1) positioned in the 1133 to $1143 \mathrm{~kb}$ region were orthologous to an Ae. tauschii gene, AEGT A20985; additionally, Bradi5g01280.1 at $1186 \mathrm{~kb}$ was orthologous to AEGTA28084 in scaffold 6859. The locations of two Ae. tauschii genes, AEGTA20985 and AEGTA28084, were 3 and $3.9 \mathrm{cM}$, respectively, distal to $I w 2$ (Figure 3); therefore, the distal part of $I w 2$ showed chromosomal synteny to Brachypodium chromosome 5. Thus, the Iw 2 chromosomal region on 2DS was generally syntenic to Brachypodium chromosome 5. However, putative orthologs of the Ae. tauschii genes from scaffold 43829 
Table 2 Colinearity between Ae. tauschii and Brachypodium in the syntenic genomic regions around Iw2

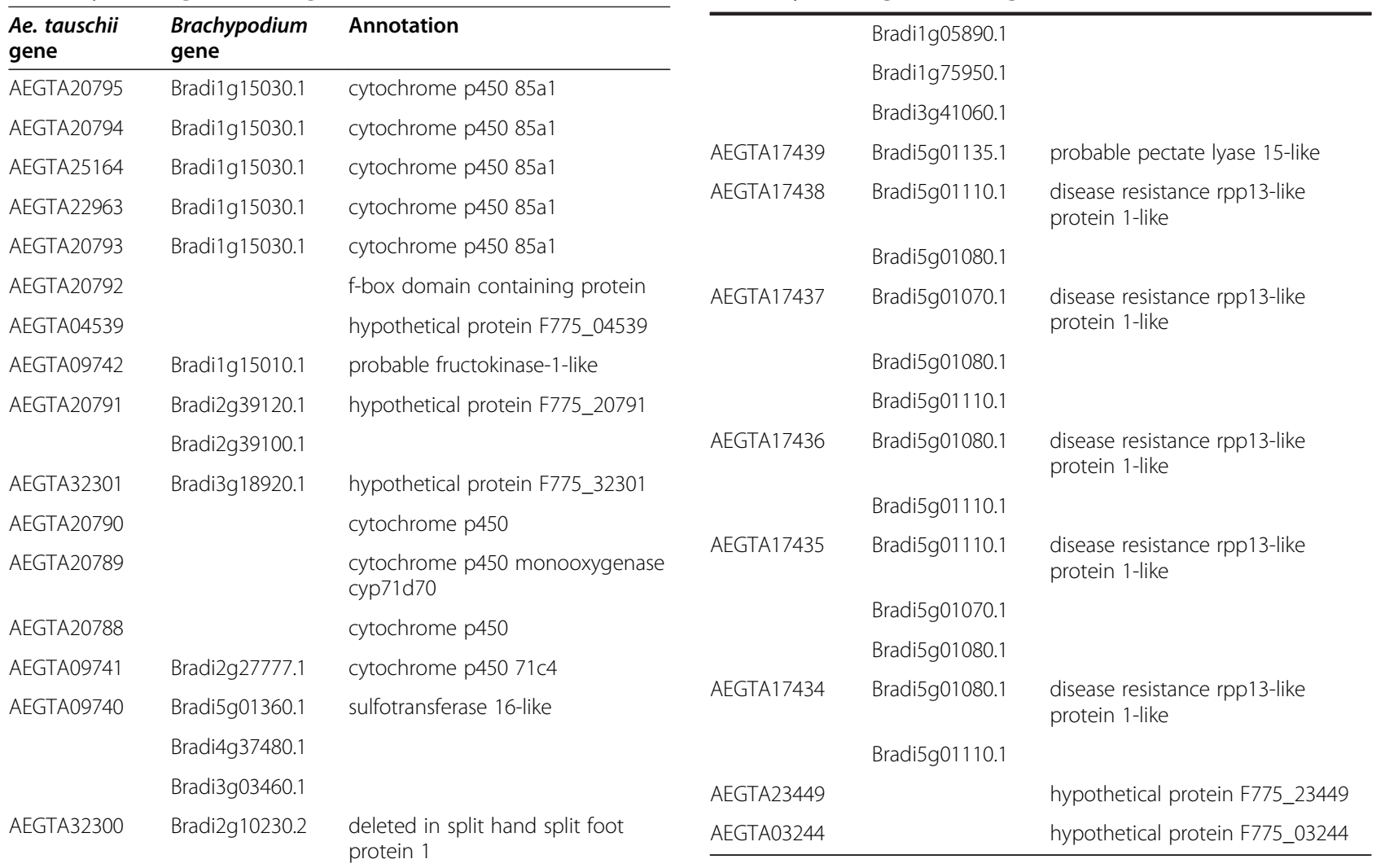

Bradi2g10230.1

AEGTA24906 Bradi5g01180.1 brown planthopper-induced resistance protein 1

AEGTA19771 Bradi3g02290.1 laccase-15-like

Bradi3g02300.1

Bradi3g02370.1

Bradi4g11840.1

AEGTA19772 Bradi4g36820.1

agmatine coumaroyltransferase-2like

Bradi3g02310.1

Bradi4g36850.1

AEGTA43098

AEGTA33234

Bradi5g01167.1

AEGTA19773

AEGTA09277

AEGTA17544

Bradi5g01167.1

protein da1-related 1-like

AEGTA08264

Bradi5g01160.1

AEGTA03281

AEGTA17543

Bradi1g30630.1

Bradi3g46930.1

Bradi5g12460.1

AEGTA17542 Bradi1g33650.1 disease resistance protein rpm1

I-type lectin-domain containing receptor kinase -like

cytochrome p450 84a1

disease resistance protein rpm1

protein da1-related 1-like

cell number regulator 2-like

cell number regulator 2-like

serine threonine-protein kinase receptor
Table 2 Colinearity between Ae. tauschii and Brachypodium in the syntenic genomic regions around Iw2 (Continued)

were assigned to Brachypodium chromosomes 1 and 2 . Two paralogous Ae. tauschii genes, AEGTA19771 and AEGTA19772, on scaffold 10812 were orthologous to three paralogous Brachypodium genes (Bradi3g02290.1, Bradi3g02300.1, and Bradi3g02370.1) on Brachypodium chromosome 3 . Therefore, the chromosomal synteny between Ae. tauschii and Brachypodium around the Iw 2 orthologs was complex with regard to chromosome structure.

To determine the genetic associations among the developed markers and glaucousness, 13 Iw2-linked PCR markers-including five CAPSs, five SSRs, one HRM, one insertion/deletion (indel), and one dominant (presence or absence) marker-were used to genotype the $210 \mathrm{Ae}$. tauschii accessions (Table 3). For eight of the 13 markers, the 210 accessions exhibited just two apparent alleles; additionally, the set of accessions exhibited just three distinct electrophoresis patterns-including the KU-2154type, the KU-2126- type, and one other type-at one SSR marker for WE6. The other four SSR markers were highly polymorphic among the accessions; specifically, each marker gave rise to more than three distinct electrophoresis patterns.

\section{Iw2-linked marker genotypes in Ae. tauschii}


Table 3 Association between Iw2-linked marker genotypes and glaucous versus non-glaucous phenotypes in 210 accessions of Ae. tauschii and the distribution of marker genotypes among Iranian wheat landraces

\begin{tabular}{|c|c|c|c|c|c|c|c|c|c|c|}
\hline \multirow[t]{2}{*}{$\begin{array}{l}\text { Marker } \\
\text { name }\end{array}$} & \multirow[t]{2}{*}{$\begin{array}{l}\text { Marker } \\
\text { type }\end{array}$} & \multirow[t]{2}{*}{$\begin{array}{l}\text { No. } \\
\text { accessions }\end{array}$} & \multicolumn{3}{|c|}{$\begin{array}{l}\text { Glaucous phenotype } \\
(N=20)\end{array}$} & \multicolumn{3}{|c|}{$\begin{array}{l}\text { Non-glaucous } \\
\text { phenotype }(N=190)\end{array}$} & \multirow{2}{*}{$\begin{array}{l}P \text {-value for } F \text {-test } \\
\text { in the association } \\
\text { analysis }^{\mathrm{a}}\end{array}$} & \multirow[t]{2}{*}{$\begin{array}{l}\text { Iranian wheat } \\
\text { landraces }(N=17\end{array}$} \\
\hline & & & $\begin{array}{l}\text { KU-2154 - } \\
\text { type }\end{array}$ & $\begin{array}{l}\text { KU-2126 - } \\
\text { type }\end{array}$ & Others & $\begin{array}{l}\text { KU-2154 - } \\
\text { type }\end{array}$ & $\begin{array}{l}\text { KU-2126 - } \\
\text { type }\end{array}$ & Others & & \\
\hline C141566873 & CAPS & 210 & 0 & 20 & 0 & 91 & 99 & 0 & 0.403 & KU-2126-type \\
\hline S43829-13 & SSR & 210 & 4 & 5 & 12 & 65 & 22 & 102 & $8.20 \mathrm{E}-05$ & Other types \\
\hline S43829-3 & CAPS & 206 & 17 & 2 & 0 & 184 & 3 & 0 & - & KU-2154-type \\
\hline S43829-12 & SSR & 207 & 0 & 6 & 14 & 136 & 37 & 14 & $1.55 \mathrm{E}-07$ & $\begin{array}{l}\text { KU-2126-type } \\
\text { (15)/Others (2) }\end{array}$ \\
\hline Xctg216249 & HRM & 210 & 9 & 11 & 0 & 177 & 13 & 0 & $1.52 \mathrm{E}-04$ & KU-2154-type \\
\hline S51038-8 & Dominant & 210 & 0 & 20 & 0 & 170 & 20 & 0 & $8.18 \mathrm{E}-10$ & KU-2154-type \\
\hline $510812-12$ & CAPS & 210 & 18 & 2 & 0 & 170 & 20 & 0 & $1.55 \mathrm{E}-05$ & KU-2126-type \\
\hline S10812-14 & Indel & 197 & 0 & 20 & 0 & 145 & 32 & 0 & $8.66 \mathrm{E}-11$ & KU-2126-type \\
\hline S10812-1 & SSR & 210 & 0 & 15 & $5^{*}$ & 135 & 0 & $55(4)$ & $3.26 \mathrm{E}-24$ & Other types \\
\hline S10812-13 & CAPS & 206 & 0 & 20 & 0 & 180 & 16 & 0 & $9.92 \mathrm{E}-16$ & KU-2126-type \\
\hline S82981-2 & SSR & 210 & 0 & 15 & $5^{*}$ & 136 & 0 & $54(5)$ & $1.95 \mathrm{E}-22$ & Other types \\
\hline WE6 & SSR & 210 & 0 & 14 & $6^{*}$ & 59 & 56 & $75(75)$ & 0.169 & Other types \\
\hline Xctg202354 & CAPS & 210 & 0 & 20 & 0 & 113 & 77 & 0 & 0.041 & KU-2126-type \\
\hline
\end{tabular}

The numbers of accessions for each genotype are represented in glaucous and non-glaucous phenotypes.

The numbers of non-glaucous-type accessions showing the genotype corresponding to the other one in the glaucous-type accessions are indicated in parenthesis *These accessions showed the same genotype different from KU-2154 and KU-2126.

${ }^{a}$ The values were calculated based on a mixed linear model in the TASSEL ver. 4.0 software.

The association analysis showed that four SSR markers (S43829-13, S43829-12, S10812-1, and S82981-2), an HRM marker (Xctg216249), the dominant marker (S51038-8), an indel marker (S10812-14), and two CAPS markers (S10812-12, and S10812-13), co-localized with $I w 2$ in the Ae. tauschii linkage map, were significantly $(P<1 \mathrm{E}-3)$ associated with variation in glaucousness; in contrast, the other three genotyped markers were not significantly associated with variation in glaucousness (Table 3). The CAPS marker S43829-3 was removed from this association analysis because of the low-frequency $(<0.05)$ allele. In particular, the KU-2126-type allele of the SSR locus S10812-1 was found only in 15 of the 20 glaucous accessions; moreover, none of the 190 non-glaucous accessions carried this KU-2126-type allele. The other five glaucous accessions carried a third allele of the S10812-1 locus. In 55 of the 190 non-glaucous accessions, only four carried the third allele of the S10812-1 locus, and the other 135 accessions carried different S10812-1 alleles. Of the four exceptional non-glaucous accessions that carried the third S10812-1 allele, two were KU-2104 and KU-2105, and these had each been used to generate a synthetic hexaploid wheat line Ldn/KU-2104 and Ldn/KU-2105, respectively; both synthetic lines showed the glaucous phenotype (Additional file 2). However, the phenotype of each synthetic hexaploid line (Ldn/KU-2074 and Ldn/KU2079) derived from the remaining two of the exceptional accessions (KU-2074 and KU-2079) was non-glaucous.
Therefore, phenotypic differentiation in glaucousness was almost completely explained by the allelic configuration at the S10812-1 locus in these natural populations of Ae. tauschii.

The 17 Iranian wheat landraces showed the KU-2154type alleles at S43829-3, Xctg216249, and S51038-8, whereas they exhibited the KU-2126-type alleles at C141566873, S10812-12, S10812-14, S10812-13, and Xctg202354. In addition, these landraces exhibited various genotypes that differed from the allelic combinations found in Ae. tauschii accessions at WE6 and four SSR marker loci, S43829-13, S10812-1, S82981-2 (Table 3). At S43829-12, 15 landraces showed the KU-2126-type genotype, and two exhibited other genotypes.

\section{Discussion}

Natural variation for wax production in Ae. tauschii

Glaucousness is presumably among the components of the domestication syndrome in tetraploid wheat $[5,6]$. Therefore, glaucousness was apparently a target of artificial selection during tetraploid domestication and common wheat speciation; nevertheless, whether glaucousness is an adaptive trait in wild wheat species remains unclear. Cuticular wax on plant surfaces plays an important role in reducing water loss under drought stress conditions for Arabidopsis and rice [1,4], and observations in these other species indicate that relationships between glaucousness and drought stress tolerance are tight. Presence of either 
the $I w 1$ or $I w 2$ allele greatly reduces $ß$-diketones in the wax components of plants, resulting in a non-glaucous phenotype $[3,10]$. Comparative study of glaucousness-related genes in near-isogenic lines (NILs) of a common wheat cultivar (S-615) $\left(\mathrm{BC}_{10} \mathrm{~F}_{3}\right.$ generation; [6]) demonstrates that $I w$ alleles had a negative impact on drought tolerance [3]. However, another study of $I w 1$ in a NIL $\left(\mathrm{BC}_{2} \mathrm{~F}_{3}\right.$ generation) of common wheat did not detect an association between Iw1 genotype and water-use efficiency [10].

In this study, we used a set of 210 accessions that represented the entire geographical range of Ae. tauschii to examine natural variation in wax production among Ae. tauschii, and found 20 glaucous accessions that were collected in the area that spans from Transcaucasus to the southern-eastern coastal region of the Caspian Sea (Figure 1, Additional file 2). In a previous study of 176 Ae. tauschii accessions collected from 105 different habitats throughout Afghanistan, Pakistan, and Iran, 17 glaucous accessions were found in this same area that spans from Transcaucasus to the southern-eastern coastal region [16]. Therefore, our findings were fully consistent with previous observations.

Most glaucous accessions belonged to the TauL2 lineage (Additional file 2). TauL2 accessions derived from geographically wide-spread sites throughout the Transcaucasus/Middle East region; these sites represented the western habitats of Ae. tauschii [19]. TauL1 accessions were collected from sites widely distributed throughout the species range, and most TauL1 accessions showed a non-glaucous phenotype. Notably, one TauL1 accession (IG127015), collected in Armenia, showed a glaucous phenotype, and the collection site was located in the middle of an area where glaucous TauL2 accessions were collected (Figure 1). Genotyping data suggested that IG127015 had an Iw2 chromosomal region that was very similar to the $I w 2$ chromosomal region of the glaucous TauL2 accessions. One possible explanation for this observation is that IG127015 acquired the Iw2 chromosomal region from some glaucous individual of the TauL2 lineage. Such introgression could occur in the natural habitat where IG127015 was originally sampled and in experimental fields where the accession was propagated for several generations. Another explanation is that IG127015 became a wax producer through a de novo recessive mutation at the $I w 2$ locus; this scenario, however, is unlikely because the molecular marker genotypes in the Iw2 chromosomal region of IG127015 were largely identical to those in the $I w 2$ chromosomal region of glaucous TauL2 accessions (Table 3).

Whether the glaucous phenotype of the exceptional TauL1 accession was due to introgression of a glaucous allele from a glaucous TauL2 plant may be difficult to discern. Genome-wide marker analyses using SNP array and diversity arrays technology (DArT) systems indicated that TauL2 was clearly distinct and genetically differentiated from TauL1 $[18,19]$. This high level of differentiation indicates that the two genealogical lineages have been reproductively isolated, and that, under natural conditions, inter-lineage hybridization seems to have occurred only rarely $[17,18]$. Nevertheless, the presence of a glaucous-type TauL1 accession indicated that the hybridization between TauL1 and TauL2 might have occurred, but the number of hybridizations seems to be quite small. Further detailed study is required to clarify the past occurrence of the TauL1-TauL2 inter-lineage hybridization in Ae. tauschii.

\section{Causal loci for variation in glaucousness among Ae. tauschii} Previous studies show that, in Ae. tauschii, the causal gene for the glaucous/non-glaucous phenotypic difference is $I w 2$, and that the genotypes of glaucous and non-glaucous accessions were W2W2iw2iw2 and W2W2Iw2Iw2, respectively [5,14]. The molecular markers tightly linked to $I w 2$ were very closely associated with glaucous versus non-glaucous phenotypic difference among the 210 accessions of Ae. tauschii (Table 3). Thus, the allelic difference at the Iw2 locus was the main contributor to the phenotypic difference between the glaucous and non-glaucous accessions of Ae. tauschii (Figure 2, Table 3). In common wheat, the markers derived from Bradi5g0 1180 and Bradi5g01160 are tightly linked to $I w 2$ as well as $I w 1[10,11]$. Because the loci that control the glaucous versus non-glaucous phenotypic difference in Ae. tauschii mapped to the chromosome 2DS region where the common wheat $I w 2$ gene resides (Figure 4), the same $I w 2$ gene is likely involved in wax production in both Ae. tauschii and common wheat. Actually, although most SSR markers around the $I w 2$ region were highly polymorphic among the Ae. tauschii accessions and Iranian wheat landraces, three markers co-localized with $I w 2$ in Ae. tauschii S10812-12, S10812-14, and S10812-13; notably, each showed the KU-2126-type alleles in each of the 17 Iranian wheat landraces (Table 3). These results indicated that the Iranian wheat landraces, which exhibited the glaucous phenotype, had the $i w 2 i w 2$ genotype.

Marker order and gene order around Ae tauschii Iw2 was well conserved with those on barley chromosome 2HS and Brachypodium chromosome 5 (Figures 3 and 4). Similar chromosomal synteny between the $I w 1$ region on 2BS and Brachypodium chromosome 5 was recently reported based on mapping with common wheat populations [10,11]. In Ae. tauschii, scaffold information derived from the draft genome data were available for detailed analysis of chromosomal synteny at the Iw2 region. Chromosomal order of the selected scaffolds at Iw2 revealed the occurrence of a local inversion during divergence between barley and Ae. tauschii (Figure 3). Moreover, information of predicted genes in the scaffolds showed that putative translocations occurred during 
divergence between Brachypodium and Ae. tauschii (Figure 4). These results also indicated that several gaps existed between the Ae. tauschii scaffolds. Thus, colinearity among barley, Brachypodium and Ae. tauschii was observed in the Iw2 syntenic region, as was reported recently [11], but further screening of Ae. tauschii BAC clones may be required for construction of the complete physical map at $I w 2$.

In the genotyping analysis with Iw2-linked markers, non-glaucous accessions with the Iw2Iw2 genotype constituted the majority of all 210 accessions (Table 3 ). However, four non-glaucous accessions (KU-2074, KU-2079, KU-2104, and KU-2015) shared a genotype at $510812-1$ (the most tightly linked marker) with five glaucous accessions (IG127015, KU-2106, KU-2158, KU-2159, KU-2160), indicating that these four nonglaucous accessions may have the $i w 2 i w 2$ genotype in spite of the S10812-1 genotypes. In fact, synthetic hexaploids from hybrids between Ldn, which has the glaucous genotype (W1W1iw1iw1) [10], and two of the four non-glaucous accessions, KU-2104 and KU-2105, exhibited the glaucous phenotype (Additional file 2). In contrast, the phenotypes of all synthetic hexaploids derived from the KU-2074 and KU-2079, were nonglaucous. Accordingly, KU-2074 and KU-2079 seemed to have the Iw2Iw2 genotype even though they shared an S10812-1 genotype with the five glaucous accessions. Taken together, all this evidence indicated that $I w 2$ was the major gene that controls inhibition of wax production in Ae. tauschii.

As yet, no loss-of-function allele has been reported for $W 2$, a major wax-producing gene in Ae. tauschii. In common wheat, however, some cultivars such as Chinese Spring and Salmon carry the recessive $w 2$ allele [5]. Similarly, non-glaucous-type accessions with the $w 1$ recessive allele have been discovered among wild emmer wheat [5]. Whether the recessive loss-of-function mutation occurred at the diploid level (i.e., in Ae. tauschii) or at the hexaploid level (i.e., in T. aestivum) is not known. Further studies are required to clarify the details of the genetic mechanism that underlies the wax production in Ae. tauschii.

\section{Implication of the Iw2 variation in hexaploid wheat speciation}

Based on a comparative genic analysis among common wheat and its ancestral species, Tsunewaki [5] suggested that common wheat, which is hexaploid, is the product of a hybrid cross that took place between a glaucous cultivated emmer wheat with the genotype W1W1iw1iw1 and a glaucous wild Ae. tauschii with the genotype the W2W2iw2iw2 genotype in the mountainous region near the southwestern coastal part of the Caspian Sea. Here, we found that, of 210 Ae. tauschii accessions, only 20 had the glaucous phenotype (Additional file 2) and that a dominant allele at the Iw2 locus were responsible for expression of the non-glaucous phenotype (Table 1). Furthermore, we found that, on the basis of the molecularmarker genotypes in the $I w 2$ chromosomal region and the phenotypes of the synthetic common wheat lines, virtually all non-glaucous accessions had the Iw2Iw2 genotype (Table 3, Additional file 2). A non-glaucous accession that had the $i w 2 i w 2$ genotype was not found among the 210 accessions. This finding was notable because the double recessive $w 2 w 2 i w 2 i w 2$ genotype, if present, would have also caused the non-glaucousness phenotype. The reason for the absence of any Ae. tauschii accession with the $w 2 w 2 i w 2 i w 2$ genotype from this collection was not clear, but this fact may indicate that functional W2 alleles confer some adaptive advantage under natural conditions. Taken together, the evidence from this study was consistent with the view that glaucous Ae. tauschii individuals that had the W2W2iw2iw2 genotype were involved in the origin of hexaploid common wheat.

Previous evidence based on isozyme variations and DNA marker polymorphisms is consistent with the hypothesis that the birthplace of hexaploid wheat is within a broad area ranging from Armenia to southwestern Caspian Iran [18,40-42]. The geographic range of the parent populations of glaucous Ae. tauschii accessions was very consistent with the region postulated in this hypothesis (Figure 1). However, the Ae. tauschii subspecies-strangulata has been postulated to be the D-genome donor of common wheat [43]. Of the 210 Ae. tauschii accessions that we examined, only 12 accessions have markedly moniliform spikes, and each of these were originally collected in the southeastern coastal Caspian region [25,26]. Taxonomically, these accessions could be classified as Ae. tauschii Coss. subspecies strangulata (Eig) Tzvel. Our data demonstrated that all these strangulata accessions, which were not glaucous, had the Iw $I$ Iw 2 genotype (Figure 1). On the assumption that the ancestral Ae. tauschii had the W2W2iw2iw2 genotype, this finding may suggest that the southeastern coastal Caspian populations of Ae. tauschii subspecies strangulata do not represent the direct descendants of the ancestral populations that gave rise to hexaploid common wheat.

\section{Conclusions}

Analysis of the Iw2 locus may contribute to improve our understanding of the evolution of hexaploid wheat. Of the 210 Ae tauschii accessions, only 20 glaucous accessions were found in the area that spans from Transcaucasia to the southern coastal region of the Caspian Sea. Of the 82 synthetic wheat lines that we examined, 15 were glaucous, and each of the 67 non-glaucous lines was produced by crossing Ldn with a non-glaucous Ae. tauschii accession. Of the 15 glaucous lines, 13 were produced by crossing Ldn with glaucous Ae. tauschii accessions. The remained 
two accessions seemed to have the Iw2Iw2 genotype according to the genotyping analysis with the Iw2-linked markers. Therefore, allelic differences at the Iw2 locus on the short arm of chromosome 2D were the main contributors to the phenotypic difference between the glaucous and non-glaucous accessions of Ae. tauschii. Some molecular markers, such as S10812-1, closely linked to Iw2 were significantly associated with variation in glaucousness in Ae. tauschii. These results suggest that the D-genome donor of common wheat could have been any Ae. tauschii variant that carried the recessive $i w 2$ allele.

\section{Availability of supporting data}

The data sets supporting the results of this article are included within the article and its supplementary files.

\section{Additional files}

Additional file 1: List of primers developed in this study.

Additional file 2: List of all 20 Ae. tauschii glaucous accessions and wheat synthetics with the glaucousness phenotype in the 82 synthetic hexaploid wheat lines.

\section{Abbreviations}

CAPS: Cleaved amplified polymorphic sequence; HRM: High resolution melting; Ldn: Langdon; SNP: Single nucleotide polymorphism; SSR: Simple sequence repeat.

\section{Competing interests}

The authors declare that they have no competing interests.

\section{Authors' contributions}

RN carried out mapping and genotyping experiments and drafted the manuscript. JCMI contributed to the bioinformatics-related analysis. YM surveyed the natural variation in Ae. tauschii and revised the manuscript. ST conceived the study, acquired the funding, examined the variation in wheat synthetics, and drafted and revised the manuscript. All authors have read and approved the final manuscript.

\section{Acknowledgements}

The authors thank Drs. Koichiro Tsunewaki and Kentaro Yoshida for helpful discussions. We are grateful to Dr. Hisashi Tsujimoto for his supplying some genotyping data of the DArT markers. This work was supported by a grant from the Ministry of Education, Culture, Sports, Science and Technology of Japan (Grant-in-Aid for Scientific Research (B) No. 25292008 to ST.

\section{Author details}

'Graduate School of Agricultural Science, Kobe University, Rokkodai 1-1, Nada, Kobe 657-8501, Japan. ²Department of Bioscience, Fukui Prefectural University, Matsuoka, Eiheiji, Yoshida, Fukui 910-1195, Japan.

Received: 18 June 2014 Accepted: 10 September 2014

Published online: 16 September 2014

\section{References}

1. Seo PJ, Lee SB, Suh MC, Park MJ, Go YS, Park CM: The MYB96 transcription factor regulates cuticular wax biosynthesis under drought conditions in Arabidopsis. Plant Cell 2011, 23:1138-1152.

2. Mao B, Cheng Z, Lei C, Xu F, Gao S, Ren Y, Wang J, Zhang X, Wang J, Wu F, Guo X, Liu X, Wu C, Wang H, Wang J: Wax crystal-space leaf2, a rice homologue of WAX2/GL1, is involved in synthesis of leaf cuticular wax. Planta 2012, 235:39-52.
3. Zhang Z, Wang W, Li W: Genetic interactions underlying the biosynthesis and inhibition of ß-diketones in wheat and their impact on glaucousness and cuticle permeability. PLOS ONE 2013, 8:e54129.

4. Zhu X, Xiong L: Putative megaenzyme DWA1 plays essential roles in drought resistance by regulating stress-induced wax deposition in rice. Proc Natl Acad Sci U S A 2013, 110:17790-17795.

5. Tsunewaki K: Comparative gene analysis of common wheat and its ancestral species. II. Waxiness, growth habit and awnedness. Jpn J Bot 1966, 19:175-229.

6. Tsunewaki K, Ebana K: Production of near-isogenic lines of common wheat for glaucousness and genetic basis of this trait clarified by their use. Genes Genet Syst 1999, 74:33-41.

7. Liu Q, Ni Z, Peng H, Song W, Liu Z, Sun Q: Molecular mapping of a dominant non-glaucousness gene from synthetic hexaploid wheat (Triticum aestivum L.). Euphytica 2007, 155:71-78.

8. Yoshiya K, Watanabe N, Kuboyama T: Genetic mapping of the genes for -nonglaucous phenotype in tetraploid wheat. Euphytica 2011, 177:293-297.

9. Okamoto Y, Kajimura T, Ikeda TM, Takumi S: Evidence from principal component analysis for improvement of grain shape- and spikelet morphology-related traits after hexaploid wheat speciation. Genes Genet Syst 2012, 87:299-310.

10. Adamski NM, Bush MS, Simmonds J, Tumer AS, Mugford SG, Jones A, Findlay K, Pedentchouk N, Von Wettstein-Knowles P, Uauy C: The inhibitor of wax 1 locus (IW1) prevents formation of $ß$ - and $\mathrm{OH}$-ß-diketones in wheat cuticular waxes and maps to a sub-cM on chromosome arm 2BS. Plant J 2013, 74:989-1002.

11. Wu H, Qin J, Han J, Zhao X, Ouyang S, Liang Y, Zhang D, Wang Z, Wu Q, Xie J, Cui Y, Peng H, Sun Q, Liu Z: Comparative high-resolution mapping of the wax inhibitors IW 1 and IW2 in hexaploid wheat. PLOS ONE 2013, 8:e84691.

12. Dubcovsky J, Echaide M, Giancola S, Rousset M, Luo MC, Joppa LR, Dvorak J: Seed-storage-protein loci in RFLP maps of diploid, tetraploid, and hexaploid wheat. Theor App/ Genet 1997, 90:247-252

13. Wang J, Li W, Wang W: Fine mapping and metabolic and physiological characterization of the glume glaucousness inhibitor locus Iw3 derived from wild wheat. Theor App/ Genet 2014, 127:831-841.

14. Watanabe N, Takesada N, Shibata Y, Ban T: Genetic mapping of the genes for glaucous leaf and tough rachis in Aegilops tauschii, the D-genome progenitor of wheat. Euphytica 2005, 144:119-123.

15. Simmonds JR, Fish LJ, Leverington-Waite MA, Wang Y, Howell P, Snape JW: Mapping of a gene (Vir) for non-glaucous, viridescent phenotype in bread wheat derived from Triticum dicoccoides, and its association with yield variation. Euphytica 2008, 159:333-341.

16. Kihara H, Tanaka M: Morphological and physiological variation among Aegilops squarrosa strains collected in Pakistan, Afghanistan and Iran. Preslia 1958, 30:241-251.

17. Mizuno N, Yamasaki M, Matsuoka Y, Kawahara T, Takumi S: Population structure of wild wheat D-genome progenitor Aegilops tauschii Coss: implications for intraspecific lineage diversification and evolution of common wheat. Mol Ecol 2010, 19:999-1013.

18. Wang J, Luo MC, Chen Z, You FM, Wei Y, Zheng Y, Dvorak J: Aegilops tauschii single nucleotide polymorphisms shed light on the origins of wheat D-genome genetic diversity and pinpoint the geographic origin of hexaploid wheat. New Phytol 2013, 198:925-937.

19. Matsuoka Y, Nasuda S, Ashida Y, Nitta M, Tsujimoto H, Takumi S, Kawahara $\mathrm{T}$ : Genetic basis for spontaneous hybrid genome doubling during allopolyploid speciation of common wheat shown by natural variation analyses of the paternal species. PLOS ONE 2013, 8:e68310.

20. Jia J, Zhao S, Kong X, Li Y, Zhao G, He W, Appels R, Pfeifer M, Tao Y, Zhang $X$, Jing R, Zhang C, Ma Y, Gao L, Gao C, Spannagl M, Mayer KFX, Li D, Pan S, Zheng F, Hu Q, Xia X, Li J, Liang Q, Chen J, Wicker T, Gou C, Kuang H, He G, Luo $Y$, et al: International Wheat Genome Sequencing Consortium, Yang H, Liu X, He Z, Mao L, Wang J: Aegilops tauschii draft genome sequence reveals a gene repertoire for wheat adaptation. Nature 2013, 496:91-95.

21. Luo MC, Gu YQ, You FM, Deal KR, Ma Y, Hu Y, Huo N, Wang Y, Wang J, Chen S, Jorgensen CM, Zhang Y, McGuire PE, Pasternak S, Stein JC, Ware D, Kramer M, McCombie WR, Kianian SF, Martis MM, Mayer KFX, Sehgal SK, Li W, Gill BS, Bevan MW, Šimková H, Doležel J, Weining S, Lazo GR, Anderson $\mathrm{OD}$, et al: A 4-gigabase physical map unlocks the structure and evolution of the complex genome of Aegilops tauschii, the wheat D-genome progenitor. Proc Natl Acad Sci U S A 2013, 110:7940-7945. 
22. Takumi S, Koyama K, Fujiwara K, Kobayashi F: Identification of a large deletion in the first intron of the Vrn-D1 locus, associated with loss of vernalization requirement in wild wheat progenitor Aegilops tauschii Coss. Genes Genet Syst 2011, 86:183-195.

23. Matsuoka $Y$, Takumi $S$, Kawahara $T$ : Natural variation for fertile triploid $F_{1}$ formation in allohexaploid wheat speciation. Theor Appl Genet 2007, 115:509-518.

24. Matsuoka $Y$, Takumi S, Kawahara T: Flowering time diversification and dispersal in central Eurasian wild wheat Aegilops tauschii Coss: genealogical and ecological framework. PLOS ONE 2008, 3:e3138.

25. Matsuoka Y, Nishioka E, Kawahara T, Takumi S: Genealogical analysis of subspecies divergence and spikelet-shape diversification in central Eurasian wild wheat Aegilops tauschii Coss. Plant Syst Evol 2009, 279:233-244.

26. Takumi S, Naka Y, Morihiro H, Matsuoka Y: Expression of morphological and flowering time variation through allopolyploidization: an empirical study with 27 wheat synthetics and their parental Aegilops tauschii accessions. Plant Breed 2009, 128:585-590.

27. Kajimura T, Murai $K$, Takumi S: Distinct genetic regulation of flowering time and grain-filling period based on empirical study of $D$ genome diversity in synthetic hexaploid wheat lines. Breed Sci 2011, 61:130-141.

28. Lander ES, Green P, Abrahamson J: MAPMAKER: an interactive computer package for constructing primary genetic linkage maps of experimental and natural populations. Genomics 1987, 1:174-181.

29. Kosambi DD: The estimation of map distance from recombination values. Ann Eugen 1944, 12:172-175.

30. Beales J, Turner A, Griffiths S, Snape JW, Laurie DA: A pseudo-response regulator is misexpressed in the photoperiod insensitive Ppd-D1a mutant of wheat (Triticum aestivum L.). Theor Appl Genet 2007, 115:721-733.

31. lehisa JCM, Shimizu A, Sato K, Nasuda S, Takumi S: Discovery of highconfidence single nucleotide polymorphisms from large-scale de novo analysis of leaf transcripts of Aegilops tauschii, a wild wheat progenitor. DNA Res 2012, 19:487-497.

32. lehisa JCM, Shimizu A, Sato K, Nishijima R, Sakaguchi K, Matsuda R, Nasuda S, Takumi S: Genome-wide marker development for the wheat D genome based on single nucleotide polymorphisms identified from transcripts in the wild wheat progenitor Aegilops tauschii. Theor Appl Genet 2014, 127:261-271.

33. International Barley Genome Sequencing Consortium, Mayer KF, Waugh R, Brown JW, Schulman A, Langridge P, Platzer M, Fincher GB, Muehlbauer GJ, Sato K, Close TJ, Wise RP, Stein N: A physical, genetic and functional sequence assembly of the barley genome. Nature 2012, 491:711-716.

34. Kofler R, Schlötterer C, Lelley T: SciRoKo: a new tool for whole genome microsatellite search and investigation. Bioinformatics 2007, 23:1683-1685.

35. Matsuda R, lehisa JCM, Takumi S: Application of real-time PCR-based SNP detection for mapping of Net2, a causal D-genome gene for hybrid necrosis in interspecific crosses between tetraploid wheat and Aegilops tauschii. Genes Genet Syst 2012, 87:137-143.

36. The International Brachypodium Initiative: Genome sequencing and analysis of the model grass Brachypodium distachyon. Nature 2010, 463:763-768.

37. Bradbury PJ, Zhang Z, Kroon DE, Casstevens TM, Ramdoss Y, Buckler ES: TASSEL: software for association mapping of complex traits in diverse samples. Bioinformatics 2007, 23:2633-2635.

38. Pritchard JK, Stephens M, Donnelly P: Inference of population structure using multilocus genotype data. Genetics 2000, 155:945-959.

39. Mayer KFX, Martis M, Hedley PE, Simková H, Liu H, Morris JA, Steuernagel B, Taudien S, Roessner S, Gundlach H, Kubaláková M, Suchánková P, Murat F, Felder M, Nussbaumer T, Graner A, Salse J, Endo T, Sakai H, Tanaka T, Itoh T, Sato K, Platzer M, Matsumoto T, Scholz U, Dolezel J, Waugh R, Stein N: Unlocking the barley genome by chromosomal and comparative genomics. Plant Cell 2011, 23:1249-1263.

40. Nakai Y: Isozyme variations in Aegilops and Triticum, IV. The origin of the common wheats revealed from the study on esterase isozymes in synthesized hexaploid wheats. Jpn J Genet 1979, 54:175-189.
41. Nishikawa K, Furuta Y, Wada T: Genetic studies on alpha-amylase isozymes in wheat. III. Intraspecific variation in Aegilops squarrosa and birthplace of hexaploid wheat. Jpn J Genet 1980, 55:325-336.

42. Dvorak J, Luo MC, Yang ZL, Zhang HB: The structure of the Aegilops tauschii genepool and the evolution of hexaploid wheat. Theor Appl Genet 1998, 97:657-670.

43. Jaaska V: Aspartate aminotransferase and alcohol dehydrogenase isoenzymes: intraspecific differentiation in Aegilops tauschii and the origin of the D genome polyploids in the wheat group. Plant Syst Evol 1981, 137:259-273.

doi:10.1186/s12870-014-0246-y

Cite this article as: Nishijima et al:: The cuticular wax inhibitor locus Iw2 in wild diploid wheat Aegilops tauschii: phenotypic survey, genetic analysis, and implications for the evolution of common wheat. BMC Plant Biology 2014 14:246.

\section{Submit your next manuscript to BioMed Central and take full advantage of:}

- Convenient online submission

- Thorough peer review

- No space constraints or color figure charges

- Immediate publication on acceptance

- Inclusion in PubMed, CAS, Scopus and Google Scholar

- Research which is freely available for redistribution

Submit your manuscript at www.biomedcentral.com/submit
C Biomed Central 